\title{
Feedback control in a collective flashing ratchet
}

\author{
Francisco J. Cao ${ }^{1,2}$, Luis Dinis ${ }^{1}$ and Juan M.R. Parrondo ${ }^{1}$ \\ 1 Grupo Interdisciplinar de Sistemas Complejos (GISC) and Departamento de Fúsica Atómica, \\ Nuclear y Molecular. Universidad Complutense de Madrid. E-28040 Madrid, Spain. \\ 2 Observatoire de Paris, LERMA. 61, Avenue de l'Observatoire, \\ 75014 Paris, France. Laboratoire Associé au CNRS UMR 8112.
}

\begin{abstract}
An ensemble of Brownian particles in a feedback controlled flashing ratchet is studied. The ratchet potential is switched on and off depending on the position of the particles, with the aim of maximizing the current. We study in detail a protocol which maximizes the instant velocity of the center of mass of the ensemble at any time. This protocol is optimal for one particle and performs better than any periodic flashing for ensembles of moderate size, whereas is defeated by a random or periodic switching for large ensembles.
\end{abstract}

PACS numbers: 05.40.-a, 02.30.Yy

Rectification of thermal fluctuations is becoming a major research topic in non equilibrium statistical mechanics, with potential applications in biology, condensed matter, and nanotechnology [1, 2].

Most of these rectifiers or Brownian ratchets work by introducing an external time-dependent perturbation in an asymmetric equilibrium system. In the case of rocking ratchets, the perturbation is an AC uniform field, whereas for the flashing ratchet 3,4$]$ the perturbation consists of switching on and off an asymmetric sawtooth potential, such as the one depicted in Fig. 11 (left).

The models and applications studied so far have been focused on periodic or random time-dependent perturbations 11. On the other hand, in this Letter we study a feedback controlled perturbation, i.e., an external force depending on the state of the system. Introducing control in Brownian ratchets is relevant for the aforementioned applications. Feedback control could be implemented in systems where particles are monitored, as occurs in some experimental setups with colloidal particles [5]. Control theory is also of extreme relevance in biology [ 6,7$]$, and most protein motors probably operate as control systems (see 8] for a specific example). Finally, controlled ratchets are relevant from a theoretical point of view. The idea of rectifying thermal noise was originally introduced by Smoluchowski 9] and Feynmann [10 in relation to the Maxwell demon. Recently, Touchette and Lloyd have pointed out that the original Maxwell demon can be considered a feedback control system and found that thermodynamics imposes some limitations to control [11].

Using controlled Brownian ratchets one can build models to check these limitations and understand how information can be used to increase the performance of a system. For instance, it is not hard to prove that some single-particle ratchets become Maxwell demons if information on the position of the Brownian particle is available [12]. In this Letter we will study the more involved problem of a collective flashing ratchet, focusing on the induced current of particles.

Consider a flashing ratchet [3, 4] consisting of a Brow- nian particle in an asymmetric sawtooth potential, such as the one depicted in Fig. 1 (left). If the potential is flashed in a periodic or random way, the particle exhibits a systematic motion to the right [1, 3, 4]. However, if we know where the particle is at any time, there is a better switching strategy. We could switch on the potential whenever the force is positive and switch it off if the force is negative. Under this switching protocol, the particle feels the effective non periodic potential depicted in Fig. 11 (right). Obviously, the particle moves to the right and this is the optimal switching policy, inducing larger currents than any periodic or random switching.
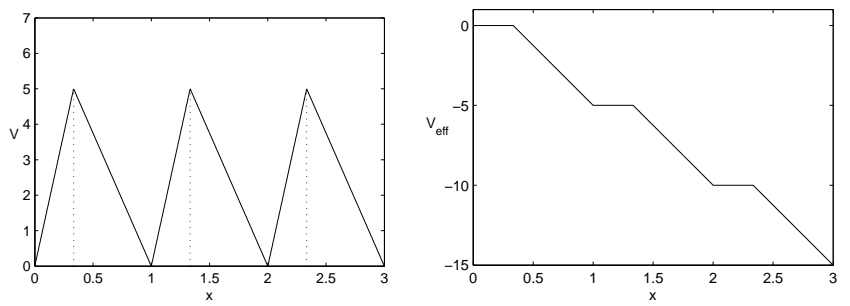

FIG. 1: The ratchet potential (left) and the corresponding effective potential for the one-particle controlled ratchet (right), both for $V_{0}=5 k T$ and $a=1 / 3$. (Units $L=1, D=1$ and $k T=1$.)

The problem of control becomes less trivial for ratchets consisting of many particles. Control strategies can induce an effective coupling among particles and the system becomes a coupled Brownian motor 13.

Consider an ensemble of $N$ overdamped Brownian particles at temperature $T$ in an external asymmetric periodic potential $V(x)$, that can be either on or off. The dynamics is described by the Langevin equation:

$$
\gamma \dot{x}_{i}(t)=\alpha(t) F\left(x_{i}(t)\right)+\xi_{i}(t) ; \quad i=1 \ldots N,
$$

where $x_{i}(t)$ is the position of particle $i, \gamma$ is the friction coefficient and $\xi_{i}(t)$ are thermal noises with zero mean and correlation $\left\langle\xi_{i}(t) \xi_{j}\left(t^{\prime}\right)\right\rangle=2 \gamma k T \delta_{i j} \delta\left(t-t^{\prime}\right)$. The force 
is given by $F(x)=-V^{\prime}(x)$ where $V(x)$ is a periodic, $V(x+L)=V(x)$, asymmetric potential defined by

$$
V(x)= \begin{cases}\frac{V_{0}}{a} \frac{x}{L} & \text { if } 0 \leq \frac{x}{L} \leq a \\ -\frac{V_{0}}{(1-a)}\left(\frac{x}{L}-a\right)+V_{0} & \text { if } a \leq \frac{x}{L} \leq 1\end{cases}
$$

and depicted in Fig. 1 left. Finally, $\alpha(t)$ is a control parameter which we assume that can take on the values 1 and 0 , i.e., the only allowed operations on the Brownian motor consist of switching on and off the potential $V(x)$.

We will consider the following two switching strategies:

- Periodic switching: $\alpha(t+\tau)=\alpha(t)$, with $\alpha(t)=1$ for $t \in[0, \tau / 2)$, and $\alpha(t)=0$ for $t \in[\tau / 2, \tau)$. This case is equivalent to the periodic flashing ratchet [3, 4], since particles are independent.

- Controlled switching:

$$
\alpha(t)=\Theta(f(t)) \quad \text { with } \quad f(t)=\frac{1}{N} \sum_{i=1}^{N} F\left(x_{i}(t)\right),
$$

where $f(t)$ is the net force per particle and $\Theta(y)$ is the Heaviside function, $\Theta(y)=1$ if $y \geq 0$ and 0 otherwise. As can be deduced from Eq. (1), this strategy maximizes the instant velocity of the center of mass, $\dot{x}_{c m}(t)=\frac{1}{N} \sum_{i=1}^{N} \dot{x}_{i}(t)$. Particles are no longer independent, due to the feedback control $\Theta(f(t))$.

We first compare numerical results for the two switching strategies described above. In Fig. 2 the center of mass velocity is plotted as a function of $N$ for the periodic switching with the optimal period [1, 3, 4, 14], and for the controlled switching, both for $V_{0}=5 k T$ and $a=1 / 3$. For this potential, the optimal period has been found to be $\tau_{\text {opt }} \simeq 0.05 L^{2} / D$, giving $\left\langle\dot{x}_{c m}\right\rangle \simeq 0.29 D / L$. The controlled switching yields a higher velocity than the periodic strategy only up to a certain $N(N \simeq 1300$ for the previous values of the parameters). We also see from simulations that the velocity goes to zero when $N \rightarrow \infty$, in the controlled case. This is surprising at first sight, because the controlled strategy given by (3) maximizes the instant velocity $\dot{x}_{\mathrm{cm}}(t)$. However, this local maximization does not ensure good results in the long term, as we have already shown for the so-called paradoxical games and other deterministic systems [15].

The case $N=1$ can be solved analytically, since it consists of a single particle moving in the effective potential depicted in the rightmost plot of Fig. 1 as mentioned above. The corresponding stationary FokkerPlanck equation can be solved, yielding

$$
\langle\dot{x}(t)\rangle_{\mathrm{st}}=\frac{2 D V_{0}\left(1-e^{\frac{-V_{0}}{k T}}\right) / L}{2 k T\left(1-a^{2}\right)\left(1-e^{\frac{-V_{0}}{k T}}\right)+V_{0} a^{2}\left(1+e^{\frac{-V_{0}}{k T}}\right)},
$$

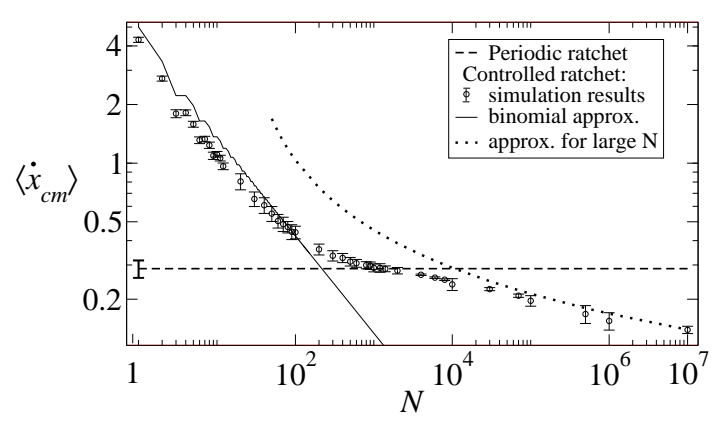

FIG. 2: Average of the speed of the center of mass, $\left\langle\dot{x}_{\mathrm{cm}}\right\rangle$, for simulations of the periodic switching with optimal period $\tau_{\mathrm{opt}}=0.05 L^{2} / D$ (dashed line); simulations of the controlled ratchet (circles with error bars); the binomial approximation, Eq. (6), (solid line); and the large $N$ approximation, Eq. (12), (dotted line); all for $V_{0}=5 k T$ and $a=1 / 3$. (Units $L=1$, $D=1$ and $k T=1$.)

where $D=k T / \gamma$ is the diffusion coefficient. For $V_{0}=$ $5 k T$ and $a=1 / 3$, this yields a stationary speed $\langle\dot{x}\rangle_{s t} \simeq$ $4.27 \mathrm{D} / \mathrm{L}$, more than 10 times larger than the highest speed obtained with a periodic switching. For $N=1$, in fact, this controlled switching is clearly optimal.

For arbitrary $N$, by summing and averaging the Langevin equations (11) with the prescription (3), one obtains the following exact equation for the mean velocity of the center of mass:

$$
\left\langle\dot{x}_{\mathrm{cm}}(t)\right\rangle=\frac{1}{\gamma}\langle\Theta(f(t)) f(t)\rangle .
$$

The net force per particle $f(t)$ can be written in terms of the number $n(t)$ of particles in the interval $[0, a L]$. Notice also that the system reaches a stationary regime, because there is not any explicit dependence on time in the controlled switching case.

The average in the r.h.s. of Eq. (5) can be approximately computed in the stationary regime making the following two assumptions: (i) particles are statistically independent (i.e., the stationary distribution has the form $\left.\rho_{s t}\left(x_{1}, x_{2}, \ldots, x_{N}\right)=\prod_{i=1}^{N} \rho_{s t}\left(x_{i}\right)\right)$ and (ii) the probability that a given particle is in the interval $[0, a L]$ is $a$. These are reasonable assumptions since both are fulfilled by the two equilibrium distributions corresponding to respectively switching on and off for a long period of time. Under this approximation, the probability distribution of the random variable $n(t)$ is a binomial distribution in the stationary regime and the average velocity becomes

$$
\left\langle\dot{x}_{\mathrm{cm}}\right\rangle_{\mathrm{st}} \simeq \frac{V_{0}}{\gamma L N} \sum_{n<N a}^{N}\left(-\frac{n}{a}+\frac{N-n}{1-a}\right)\left(\begin{array}{c}
N \\
n
\end{array}\right) a^{n}(1-a)^{N-n} .
$$

This binomial approximation, Eq. (6), has been tested for various values of $V_{0}$ and $a$ giving good results for small $N$ (see Fig. (2). Eq. (6) is in fact exact for $N=1$ in 
the limit $V_{0} /(k T) \rightarrow 0$ [as can be proven from Eq. (4)]. This is expected since the equilibrium distribution is then almost uniform both for the on and off potentials. As a consequence, the binomial approximation gives better results when decreasing $V_{0} /(k T)$, not only for $N=1$, but also for $N>1$.

For sufficiently large values of $N(\gtrsim 10)$, the binomial distribution can be approximated by a Gaussian, yielding

$$
\left\langle\dot{x}_{\mathrm{cm}}\right\rangle_{\mathrm{st}} \simeq \frac{1}{\sqrt{2 \pi \sigma^{2}}} \int_{-\infty}^{\infty} d f \Theta(f) \frac{f}{\gamma} e^{-\frac{f^{2}}{2 \sigma^{2}}}=\frac{\sigma}{\gamma \sqrt{2 \pi}},
$$

with $\sigma^{2}=V_{0}^{2} /\left[L^{2} a(1-a) N\right]$.

One can study within this approximation the following general mixed strategy depending on $f(t)$ : the potential is switched on with a probability $p_{\text {on }}(f(t))$, and switched off with probability $1-p_{\text {on }}(f(t))$. Under the gaussian approximation, the stationary velocity for this protocol is the same as in Eq. (7) but replacing $\Theta(f)$ by $p_{\text {on }}(f)$. Then it can be easily seen that the gaussian approximation predicts that the optimal strategy is the one considered in this paper, i.e., $p_{\text {on }}(f)=\Theta(f)$. However, this is not true for large $N$ (see Fig. (2).

The binomial and the gaussian approximations fail in other respects for large values of $N$. Both give $\left\langle\dot{x}_{\mathrm{cm}}\right\rangle_{\mathrm{st}} \sim$ $1 / \sqrt{N}$, whereas numerical simulations show that $\left\langle\dot{x}_{\mathrm{cm}}\right\rangle_{\mathrm{st}}$ decays more slowly.
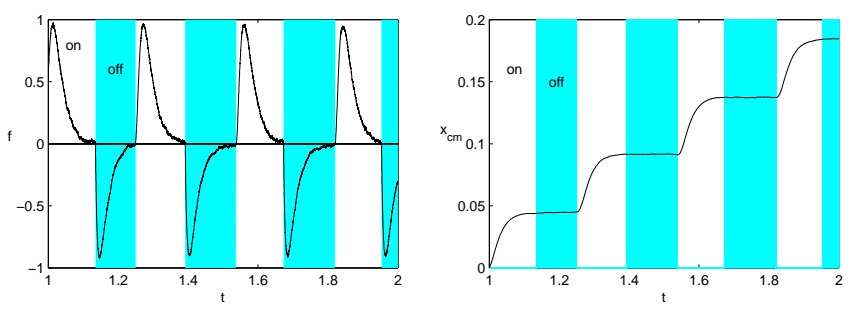

FIG. 3: $N=10^{6}$ : Time dependence of the net force per particle $f(t)$ (left) and the average position of the center of mass $\left\langle x_{\mathrm{cm}}(t)\right\rangle$ (right). Colored background indicates the offpotential time intervals. Both figures are for $V_{0}=5 k T, a=$ 1/3. (Units: $L=1, D=1$ and $k T=1$.)

For large $N$, numerical simulations reveal that the system gets trapped near the equilibrium distribution of either the on or the off potential, and switches are induced only by fluctuations of the net force per particle $f(t)$. This behavior is shown in Fig. [3 where the evolution of $f(t)$ and $\left\langle x_{\mathrm{cm}}(t)\right\rangle$ is depicted for $N=10^{6}$. These fluctuations decrease with $N$, then so does the frequency of switches, yielding $\left\langle\dot{x}_{\mathrm{cm}}\right\rangle \rightarrow 0$ for $N \rightarrow \infty$. Therefore, the system almost reaches the equilibrium distribution of each dynamics. These equilibrium distributions for the on and off potential are, respectively, $\rho_{\mathrm{st}, \text { on }}(x)=e^{-V(x) / k T} / Z$ and $\rho_{\mathrm{st}, \text { off }}(x)=1 / L$, where $Z$ is a normalization constant.
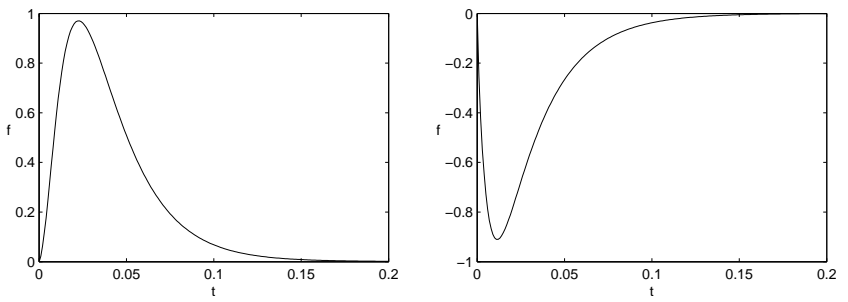

FIG. 4: $N=\infty$ : Net force per particle $f^{\infty}(t)=\langle F(x)\rangle$ as a function of time, for $V_{0}=5 k T$, and $a=1 / 3$, and for the two cases: Left: $\alpha(t)=1$ (potential on) and initial condition given by the equilibrium profile for a flat potential, i.e., $\rho_{\text {st,off }}(x)$; and right: $\alpha(t)=0$ (potential off) and initial condition given by the equilibrium profile for a ratchet potential, i.e., $\rho_{\text {st,on }}(x)$. (Units $L=1, D=1$, and $k T=1$.)

To gain some quantitative insight, let us consider the case $N=\infty$ (no fluctuations). In this case, the distribution of particles coincides with the solution of the following mean-field Fokker-Planck equation:

$$
\gamma \partial_{t} \rho(x, t)=\left[-\alpha(t) \partial_{x} F(x)+k T \partial_{x}^{2}\right] \rho(x, t),
$$

where $\alpha(t)=\Theta\left(f^{\infty}(t)\right), f^{\infty}(t) \equiv\langle F(x)\rangle$, and the average $\langle$.$\rangle is taken over \rho(x, t)$.

We have studied the evolution of $\rho(x, t)$ in two situations: (i) $\alpha(t)=1$ (potential on) with initial condition given by $\rho_{\text {st,off }}(x)=1 / L$ and (ii) $\alpha(t)=0$ (potential off) with initial condition given by $\rho_{\text {st,on }}(x)$. We have calculated the average force $f^{\infty}(t)$ as a function of time by a numerical integration of the Fokker-Planck equation. The results are plotted in Fig. (4 When the potential is switched on, the particles in the negative force interval (where the force is stronger due to the asymmetry of the potential) arrive earlier at the minima. This leads to an initial increase in the net force per particle, $f^{\infty}(t)$. [A similar interpretation can be given to Fig. 团 (right).]

For large but finite $N, f(t)$ fluctuates around $f^{\infty}(t)$ (cfr. figures 3 and (4), i.e., $f(t)=f^{\infty}(t)+$ fluctuations. Due to these fluctuations, $f(t)$ crosses zero and switches are induced. In fact, for large $N$ the controlled ratchet behaves as an excitable system: fluctuations induce the switches and, after one of these switches, the system has to perform a large excursion for the force to go back to a small value, suitable for a new "excitation". This picture allows us to calculate $\left\langle\dot{x}_{\mathrm{cm}}\right\rangle$ for large $N$. For $N$ finite, the fluctuations of $f(t)$ are of order $\sqrt{\left\langle F^{2}\right\rangle_{\text {eq }} / N}$. Therefore, a switch is possible whenever

$$
\left|f^{\infty}(t)\right| \sim \sqrt{\frac{\left\langle F^{2}\right\rangle_{\mathrm{eq}}}{N}} .
$$

In our case, fluctuations are the same for the on and off situations: $\left\langle F^{2}\right\rangle_{\mathrm{eq}}=V_{0}^{2} /\left[L^{2} a(1-a)\right]$.

On the other hand, $f^{\infty}(t)$ departs rapidly from zero, reaches a maximum and finally exhibits an exponential 
decay, both for the on and off cases (Fig. U left and right, respectively). Therefore, in each case

$$
f_{\text {on }, \text { off }}^{\infty}(t)=C_{\text {on }, \text { off }} e^{-\lambda_{\text {on }, \text { off }}\left(t-\tau_{\text {on }, \text { off }}\right)},
$$

where $C_{\mathrm{on}, \text { off }}$ are constants, $\tau_{\mathrm{on}, \text { off }}$ are the transient times of each dynamics, and $1 / \lambda_{\text {on,off }}$ are the characteristic times of the corresponding exponential decays. Combining Eqs. (9) and (10), one obtains the following switching time:

$$
t_{\mathrm{on}, \mathrm{off}} \simeq c_{\mathrm{on}, \mathrm{off}}+\frac{\ln N}{2 \lambda_{\mathrm{on}, \mathrm{off}}}
$$

where $c_{\mathrm{on} \text {,off }}$ are constants depending on the transient regime of each dynamics.

Finally, the center of mass only moves when the potential is on, covering a distance $\Delta x_{\mathrm{on}}$, as shown in Fig. 3 (right), (when the potential is off, the evolution is purely diffusive and $\Delta x_{\text {off }}=0$.) Thus, the average velocity of the particles is

$$
\left\langle\dot{x}_{\mathrm{cm}}\right\rangle=\frac{\Delta x_{\mathrm{on}}}{t_{\mathrm{on}}+t_{\mathrm{off}}} \simeq \frac{\Delta x_{\mathrm{on}}}{b+d \ln N} .
$$

where $b=c_{\text {on }}+c_{\text {off }}$ and $d=\left(\lambda_{\text {on }}+\lambda_{\text {off }}\right) /\left(2 \lambda_{\text {on }} \lambda_{\text {off }}\right)$. Every parameter in Eq. (12) can be obtained from the numerical integration of the corresponding Fokker-Planck equation for $N=\infty$. We have performed such an integration for $V_{0}=5 k T$ and $a=1 / 3$, yielding $\Delta x_{\mathrm{on}}=0.047 L$, $\lambda_{\text {on }}=40 D, \lambda_{\text {off }}=39 D$ and $b=-0.071 D^{-1}$. Theory and numerical simulations show a good agreement for large values of $N$ (see Fig. 2). This good agreement has also been verified for other values of $V_{0}, a$, and for other potential shapes, for example $V(x)=V_{0}[\sin (2 \pi x / L)+$ $\sin (4 \pi x / L)]$.

It is interesting to note that the advance $\Delta x_{\mathrm{on}}$ is mainly covered during a transient time, $\tau_{\text {on }}$, that is of the order of half the optimal period for periodic switching. Longer switching periods, as we have in the present controlled ratchet for large $N$, lead to lower performance. On the other hand, for small $N$ the controlled ratchet manages to use the information provided by $f$ to do $a d$ equate faster switches and increase performance.

Notice that the feedback control studied in this Letter is itself a rectification mechanism. Hence, for $a \neq 1 / 2$, there are two sources of spatial asymmetry in our system: the feedback control and the shape of the ratchet potential. Feedback control induces a net flux even for symmetric potentials, $a=1 / 2$. In this case, $f^{\infty}(t)=0$ and one only has to consider the fluctuations in $f(t)$. Consequently, the binomial approximation works well even for large values of $N$. Introducing an asymmetry in the potential, $a<1 / 2$, favors the flow of particles for any $N$ and changes the large $N$ behavior of $\left\langle\dot{x}_{\mathrm{cm}}\right\rangle$ from $1 / \sqrt{N}$ to $1 / \ln N$ [cfr. Eqs. (17) and (12)].

Summarizing, we have computed the current induced in an ensemble of Brownian particles by a potential that is switched on and off according to a simple feedback strategy maximizing the instant velocity of the center of mass, and compared it with the current induced by a periodic switching. The results show that, for small $N$, the current is better in the controlled case than in the periodic switching, as expected. However, for large ensembles the controlled ratchet performs worse than the periodic strategy.

There are a number of open problems prompted by our work. First, finding the optimal protocol for $N>1$, which probably differs from the one considered in this Letter. Secondly, we have focused on maximizing the current, but it would be relevant to consider also the maximization of the efficiency, specially to asses the entropic value of the information about fluctuations and the limitations imposed by thermodynamics to feedback control 11].

This work has been financially supported by grants BFM2001-0291-C02-02 and BFM2003-02547/FISI from MCYT (Spain). Authors also acknowledge support from UCM (Spain) through research project PR1/03-11595, and from ESF Programme STOCHDYN.

[1] P. Reimann, Phys. Rep. 361, 57 (2002).

[2] H. Linke, Appl. Phys. A 75, 167 (2002).

[3] A. Ajdari and J. Prost, C.R. Acad. Sci. Paris II, 315, 1635 (1992).

[4] R.D. Astumian and M. Bier, Phys. Rev. Lett. 72, 1766 (1994).

[5] J. Rousselet, L. Salome, A. Ajdari and J. Prost, Nature 370, 446 (1994).

[6] J.H. Milsum, Biological Control Analysis (McGraw-Hill Book Company, New York, 1966).

[7] H. Kitano, Foundations of Systems Biology (MIT Press, Cambridge, 2001).

[8] P. Serwer, J. Struct. Biol. 141, 179 (2003).

[9] M.V. Smoluchowski, Phys. Z. 13, 1069 (1912).

[10] R.P. Feynman, R.B. Leighton, M. Sands, The Feynman Lectures on Physics (Addison Wesley, Reading, MA 1963).

[11] H. Touchette and S. Lloyd, Phys. Rev. Lett. 84, 1156 (2000). H. Touchette and S. Lloyd, Physica A 331, 140 (2004).

[12] J.M.R. Parrondo and B. Jiménez de Cisneros, Applied Physics A, 75, 179-191 (2002). B. Jiménez de Cisneros, P. Reimann and J.M.R. Parrondo, Europhys. Lett. 64, 599 (2003).

[13] F. Jülicher and J. Prost, Phys. Rev. Lett. 75, 2618 (1995). P. Reimann, R. Kawai and C. Van den Broeck, P. Hänggi, Europhys. Lett. 45, 545 (1999).

[14] J.M.R. Parrondo, J.M. Blanco, F.J. Cao and R. Brito, Europhys. Lett., 43, 248-254 (1998).

[15] L. Dinis and J.M.R. Parrondo, Europhys. Lett. 63, 319 (2003). L. Dinis and J.M.R. Parrondo, Inefficiency of voting in Parrondo games. Physica A. In press. 